\title{
Interpolação Cúbica Adaptativa
}

\author{
Eric Magalhães Delgado
}

Resumo-Neste artigo, um algoritmo de interpolação cúbica, que adapta o filtro de reconstrução através de propriedades locais da seqüência a ser interpolada, é proposto. A idéia é minimizar uma medida de distorção entre o filtro de reconstrução cúbico e o filtro de reconstrução ideal. Simulações em imagens típicas mostram um ganho quantitativo significativo do algoritmo adaptativo quando comparado com algoritmos clássicos.

Abstract-In this paper, an algorithm of cubic interpolation, that adapts the reconstruction filter through local properties of the sequence to be interpolated, is proposed. The idea is to minimize a measure of distortion between the cubic reconstruction filter and the ideal reconstruction filter. Simulations in typical images show a significant quantitative gain of the adaptative algorithm when compared with classic algorithms.

Palavras-Chave-Reconstrução, Interpolação, Convolução Cúbica.

\section{Introdução}

Nas operações geométricas de mudança de resolução, rotação ou translação de uma imagem é necessário aplicar conceitos relacionados à reconstrução do sinal contínuo para obtenção da imagem de saída.

A diferença essencial entre os processos de reconstrução e interpolação é que a resolução associada à reconstrução é infinita. Já o processo de interpolação possui uma resolução finita, o que viabiliza sua implementação em sistemas digitais.

Conforme será descrito na Sec. II-B, o filtro de reconstrução cúbico é, na verdade, uma família de filtros de reconstrução em virtude da existência do grau de liberdade na sua definição.

Neste artigo, é proposto um algoritmo de interpolação adaptativo que minimiza uma medida de distorção entre o filtro de interpolação ideal e o filtro de interpolação da família cúbica.

Para avaliar o desempenho do algoritmo adaptativo foram realizados testes em imagens típicas. Para esse caso particular de implementação, a imagem foi dividida em seqüências unidimensionais de forma que cada seqüência seja modelada como resultado de um sistema Auto-Regressivo - AR de $1^{\mathrm{a}}$ ordem.

\section{ReCOnstruÇÃo}

\section{A. Descrição}

Reconstrução é o processo inverso ao processo de amostragem, ou seja, é o processo de obtenção de uma função contínua $x(s)$ a partir de suas amostras $x_{k}$.

A reconstrução perfeita de um sinal $x(s)$ só é possível quando o mesmo é limitado em faixa, ou seja, $X(j \Omega)=0$ para

Eric Magalhães Delgado estava na Universidade Estadual de Campinas Unicamp. Agora está na Agência Nacional de Telecomunicações - Anatel. Email: eric.mdelgado@gmail.com
$|\Omega|>\Omega_{M}$, e o período de amostragem obedece à inequação $\mathrm{T} \leq \pi / \Omega_{M}$

Sem perdas de generalidades, serão considerados que a freqüência máxima de $x(s)$ é $\Omega_{M}=\pi \mathrm{rad} / \mathrm{s}$ e que o período de amostragem é $\mathrm{T}=1 \mathrm{~s}$.

A relação entre a função contínua $x(s)$ e suas amostras $x_{k}$ é dada em (1).

$$
x(s)=\sum_{k=-\infty}^{\infty} x[k] h_{r}(s-k)
$$

sendo $h_{r}(s)$ o filtro de reconstrução.

Como característica fundamental do processo de reconstrução, os valores do sinal $x(s)$ nas posições inteiras devem coincidir com os valores das amostras da seqüência $x_{k}$, isto é, $x(s=k)=x[k]$. Em conseqüência, o filtro de reconstrução deve possuir $h_{r}(0)=1$ e $h_{r}(\ell)=0 \forall \ell \in \mathbb{Z}^{*}$.

Observe que recontrução é um processo associado a uma resolução infinita, uma vez que é obtido um sinal contínuo na saída. No entanto, na maioria das aplicações, o objetivo é aumentar a resolução de uma sequiência $x_{k}$ por um fator finito e inteiro $L$. Na Fig. 1, está representado o processo de interpolação por um fator inteiro $L$. Inicialmente são inseridas $(L-1)$ amostras nulas entre cada amostra de $x_{k}$. A seqüência resultante $x_{e, k}$ é então filtrada para obtenção de $y_{k}$, seqüência esta associada a uma resolução $L$ vezes superior à resolução de $x_{k}$.

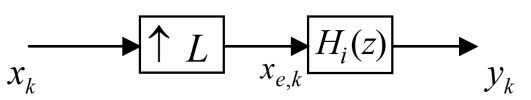

Fig. 1. Interpolação da seqüência $x_{k}$ por um fator inteiro $L$.

Uma vez que $X_{e}\left(e^{j \omega}\right)=X\left(e^{j \omega L}\right)$, a relação entre as densidades espectrais de potência das seqüências $x_{k}$ e $x_{e, k}$ é dada em (2).

$$
S_{x_{e}}\left(e^{j \omega}\right)=\frac{S_{x}\left(e^{j \omega L}\right)}{L}
$$

O filtro de interpolação $H_{i}(z)$ é uma versão discreta do filtro de reconstrução. Ou seja,

$$
h_{i}[k]=\left.h_{r}(s)\right|_{s=k \mathrm{~T}}
$$

com período de amostragem $\mathrm{T}=(1 / L)$.

\section{B. Filtro de Reconstrução Cúbico}

O filtro de reconstrução ideal é um filtro passa-baixa com ganho DC unitário e freqüência de corte $\Omega_{C}=\pi \mathrm{rad} / \mathrm{s}$. A resposta espacial do filtro de reconstrução ideal é dada em (4). 
TABELA I

CONDIÇÕES PARA OBTENÇÃO DOS COEFICIENTES DO FILTRO DE RECONSTRUÇÃO CÚBICO.

\begin{tabular}{|c|c|c|c|c|}
\hline $\begin{array}{l}\text { Interpolador: } \\
h_{C}(0)=1 \mathrm{e} h_{C}(\ell)=0, \forall \ell \in \mathbb{Z}^{*}\end{array}$ & $\begin{array}{r}\alpha_{0,0} \\
\alpha_{1,0}+\alpha_{1,1}+\alpha_{1,2}+\alpha_{1,3} \\
\alpha_{1,0}+2 \alpha_{1,1}+4 \alpha_{1,2}+8 \alpha_{1,3}\end{array}$ & $\begin{array}{l}= \\
= \\
=\end{array}$ & $\begin{array}{l}1 \\
0 \\
0\end{array}$ & $\begin{array}{l}(1) \\
(2) \\
(3)\end{array}$ \\
\hline $\begin{array}{l}\text { Ganho DC: } \\
\sum_{\ell \rightarrow-\infty}^{\infty} h_{C}\left(s_{0}+\ell\right)=1, \forall s_{0} \in \mathbb{R}\end{array}$ & $2 \sum_{i=0}^{3}\left(\frac{\alpha_{0, i}+\alpha_{1, i}}{2^{i}}\right)$ & $=$ & 1 & $(4)$ \\
\hline $\begin{array}{l}\text { Continuidade C0: } \\
\lim _{s \rightarrow s_{0}-} h_{C}(s)=\lim _{s \rightarrow s_{0}} h_{C}(s)=h_{C}\left(s_{0}\right), \forall s_{0} \in \mathbb{R}\end{array}$ & $\begin{array}{r}\alpha_{0,0}+\alpha_{0,1}+\alpha_{0,2}+\alpha_{0,3} \\
\alpha_{1,0}+2 \alpha_{1,1}+4 \alpha_{1,2}+8 \alpha_{1,3}\end{array}$ & $\begin{array}{l}= \\
=\end{array}$ & $\begin{array}{l}\alpha_{1,0}+\alpha_{1,1}+\alpha_{1,2}+\alpha_{1,3} \\
0\end{array}$ & $\begin{array}{l}(5) \\
(6)\end{array}$ \\
\hline $\begin{array}{l}\text { Continuidade C1: } \\
\lim _{s \rightarrow s_{0}-} h_{C}^{\prime}(s)=\lim _{s \rightarrow s_{0}+} h_{C}^{\prime}(s)=h_{C}^{\prime}\left(s_{0}\right), \forall s_{0} \in \mathbb{R}\end{array}$ & $\begin{array}{r}\alpha_{0,1}+2 \alpha_{0,2}+3 \alpha_{0,3} \\
\alpha_{1,1}+4 \alpha_{1,2}+12 \alpha_{1,3}\end{array}$ & $\begin{array}{l}= \\
=\end{array}$ & $\begin{array}{l}\alpha_{1,1}+2 \alpha_{1,2}+3 \alpha_{1,3} \\
0\end{array}$ & $\begin{array}{l}(7) \\
(8)\end{array}$ \\
\hline
\end{tabular}

$$
h_{B}(s)=\frac{\sin (\pi s)}{\pi s}, s \in \mathbb{R}
$$

Conforme (4), $h_{B}$ possui região de suporte infinita. Em consequiência, não é realizável. Uma aproximação usual do filtro de reconstrução ideal $h_{B}$ é obtida a partir de polinômios cúbicos no domínio $(-2,2)$. Ou seja,

$$
h_{C}(s)= \begin{cases}\alpha_{0,0}+\alpha_{0,1}|s|+\alpha_{0,2}|s|^{2}+\alpha_{0,3}|s|^{3} & \text { se }|s|<1 \\ \alpha_{1,0}+\alpha_{1,1}|s|+\alpha_{1,2}|s|^{2}+\alpha_{1,3}|s|^{3} & \text { se } 1 \leq|s|<2 \\ 0 & \text { se }|s| \geq 2\end{cases}
$$

Para obtenção dos coeficientes de $h_{C}$, supõe-se que $h_{C}$ é uma função interpoladora, contínua, possui $1^{\mathrm{a}}$ derivada contínua e ganho DC unitário. Veja a Tab. I.

Observe que são obtidas sete equações linearmente independentes, uma vez que as equações (3) e (6) da Tab. I são iguais. Seja $\alpha_{1,3}=a$. Em conseqüência,

$$
h_{C}(s)= \begin{cases}1-(a+3)|s|^{2}+(a+2)|s|^{3} & \text { se }|s|<1 \\ -4 a+8 a|s|-5 a|s|^{2}+a|s|^{3} & \text { se } 1 \leq|s|<2 \\ 0 & \text { se }|s| \geq 2\end{cases}
$$

Uma vez que o parâmetro $a$ é o grau de liberdade de $h_{C}$, o filtro de reconstrução cúbico $h_{C}$ é, na verdade, uma família de filtros de reconstrução.

A transformada de Fourier da família cúbica é dada em (7).

$$
\begin{aligned}
H_{C}(\Omega)= & -\frac{4}{\Omega^{4}}[(4 a+3) \Omega \sin \Omega+a \Omega \sin 2 \Omega+ \\
& +6(\cos \Omega-1)+3 a(\cos 2 \Omega-1)]
\end{aligned}
$$

Apesar de ser função do parâmetro $a$, na frequência de corte do filtro, $H_{C}(\Omega)$ independe de $a$, assumindo o valor de $48 / \pi^{4}$ (aproximadamente $-6 \mathrm{~dB}$ ). Veja a Fig. 2 .

Diferentes conceitos são usados para determinação do parâmetro $a$.

- Quando $-3<a<0$, as concavidades de $h_{C}$ em $s=0$ e $s=1$ são iguais às do filtro de reconstrução ideal $h_{B}$ [1].

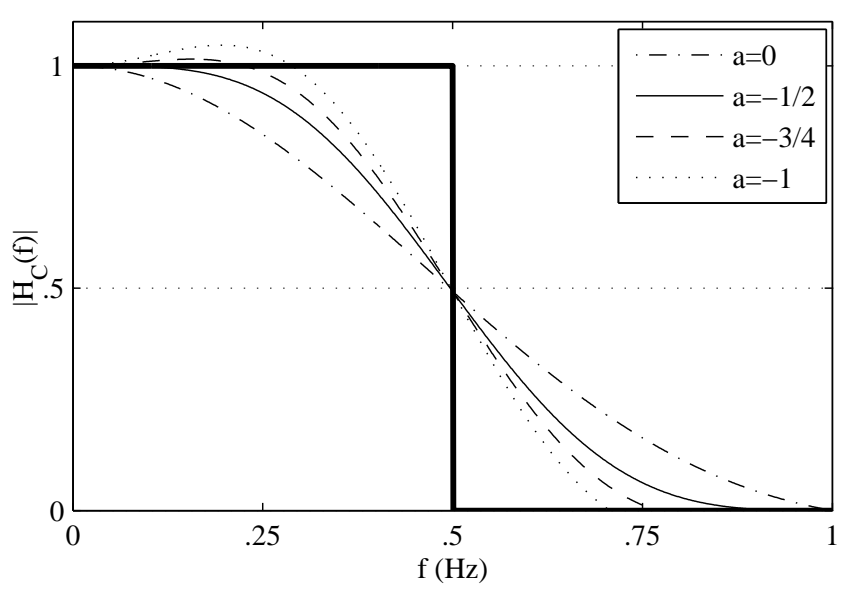

Fig. 2. Resposta espectral de $h_{C}$ para diversos valores do parâmetro $a$ no domínio $f<1 \mathrm{~Hz}$.

- Quando $a=0, h_{C}$ é associado a apenas dois pontos de suporte, ou seja, cada valor do sinal reconstruído $x(s)$ é função de apenas duas amostras da seqüência $x_{k}$. Para qualquer outro valor do parâmetro $a, h_{C}$ é associado a quatro pontos de suporte.

- Quando $a=-1 / 2, h_{C}$ reconstroi os três primeiros termos da expansão em série de Taylor do sinal $x(s)$ [2].

- Quando $a=-3 / 4, h_{C}$ possui $2^{\text {a }}$ derivada contínua.

- Quando $a=-1$, todos os coeficientes de $h_{C}$ são números inteiros. Além disso, $h_{C}$ possui taxa de decaimento em $s=1$ igual à do filtro de reconstrução ideal $h_{B}$.

Na Fig. 2 são mostradas as respostas espectrais de alguns filtros de reconstrução da família cúbica. Observe que, com a diminuição do parâmetro $a$ no intervalo $[-1,0]$, o filtro possui uma resposta espectral com energia mais concentrada nas baixas freqüências. Essa concentração pode levar ao overshoot na faixa de passagem, mas proporciona uma melhor resposta espectral na faixa de rejeição.

Para uma análise detalhada dos filtros de reconstrução ver [3-7]. 


\section{INTERPOLAÇÃo CÚBICA AdAPTATIVA}

Conforme mencionado na Sec. II-B, diferentes conceitos são usados para determinação do parâmetro $a$ da família cúbica de filtros de reconstrução. Neste contexto, é proposto o valor do parâmetro $a$ que se adapta às características da seqüência $x_{k}$ de forma a minimizar uma medida de distorção entre os filtros de interpolação $h_{C}$ e $h_{B}$.

$\mathrm{Na}$ Sec. III-A, é definida a medida de distorção a ser minimizada e, na Sec. III-B, é descrita uma possível implementação do algoritmo que adapta o parâmetro $a$ da família cúbica no processo de interpolação de sequiência $x_{k}$. Neste caso, a sequiência $x_{k}$ é modelada como resultado de um sistema AR de $1^{\text {a }}$ ordem.

Para uma análise de outros algoritmos adaptativos de interpolação cúbica ver [10-13].

\section{A. Área de Distorção}

O filtro de interpolação $H_{i}(z)$ pode ser modelado como a soma do filtro de interpolação ideal $H_{B}(z)$ e de um filtro diferença $\Delta(z)$. Veja a Fig. 3.

O módulo da resposta espectral do filtro diferença é

$$
\left|\Delta\left(e^{j \omega}\right)\right|=\left|H_{i}\left(e^{j \omega}\right)-H_{B}\left(e^{j \omega}\right)\right|
$$

Observe na Fig. 3 que a componente $y_{1, k}$ de $y_{k}$ é a seqüência desejada. Portanto, a componente $y_{2, k}$ de $y_{k}$ pode ser interpretada como o ruído associado ao processo de interpolação.

Em (9) é definida a Área de Distorção - AD.

$$
\mathrm{AD} \triangleq \frac{1}{L} \int_{\omega=-\pi}^{\pi}\left|\Delta\left(e^{j \omega}\right)\right|^{2} S_{x}\left(e^{j \omega L}\right) \mathrm{d} \omega
$$

Observe que (9) corresponde à área da densidade espectral de potência da componente $y_{2}$ no domínio $|\omega|<\pi$ rad.

$\mathrm{O}$ algoritmo adaptativo usa o valor do parâmetro $a$ que minimiza a AD para um dado fator de interpolação $L$. Ou seja,

$$
\frac{\partial \mathrm{AD}}{\partial a}=0 \Rightarrow a=a^{*}
$$

O filtro de interpolação que satisfaz (10) será referenciado como ${ }_{\left(a=a^{*}\right)} h_{C}$.

Para exemplificar o uso da $\mathrm{AD}$, considere que a seqüência a ser interpolada, $x_{k}$, é resultado de um sistema AR de $1^{\mathrm{a}}$ ordem, cuja a entrada é um ruído branco, e que a densidade espectral de potência da seqüência $x_{k}$ é dada em (11).

$$
S_{x}\left(e^{j \omega}\right)=\frac{1-\rho^{2}}{1-2 \rho \cos \omega+\rho^{2}} \sigma_{x}^{2}
$$

Na Fig. 4, está o mapeamento entre o parâmetro $a$ que minimiza a $\mathrm{AD}$ e o coeficiente de correlação $\rho$ do sistema, obtidos a partir do fator de interpolação $L=4$.

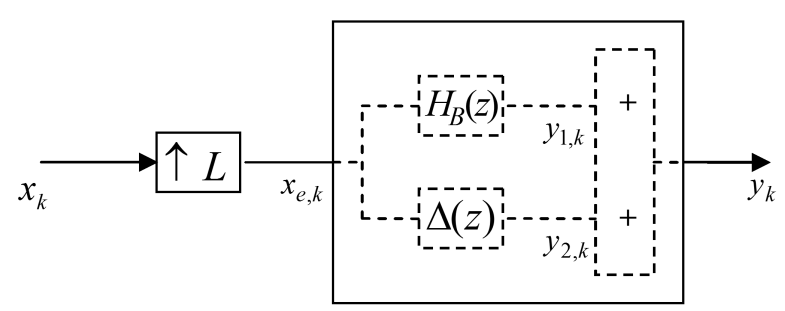

Fig. 3. Diagrama de blocos do processo de interpolação.

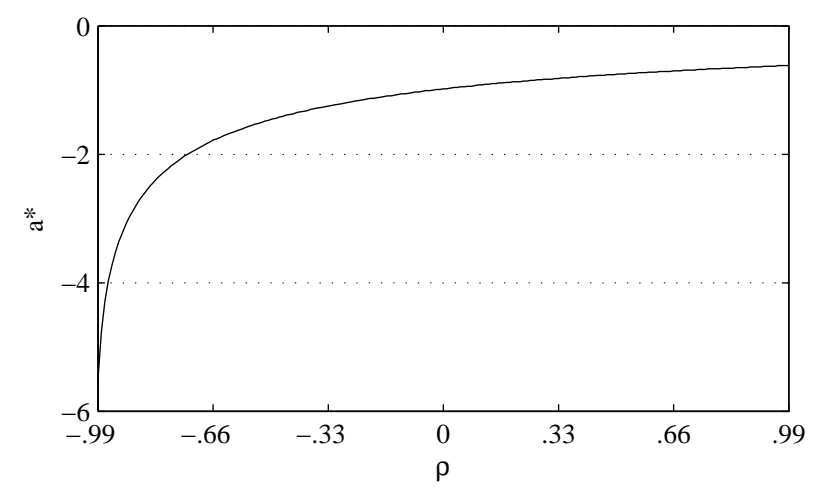

Fig. 4. Valor do parâmetro $a$ da família cúbica que minimiza a AD para um fator de interpolação $L=4$.

\section{B. Algoritmo}

O algoritmo adaptativo implementado no processo de interpolação tem como objetivo explorar a redundância espacial de uma seqüência cuja energia é concentrada nas baixas freqüências, como é o caso da maioria das imagem reais.

Regiões de uma imagem cujas variações entre pixels vizinhos ocorrem de forma suave podem ser modeladas como saídas de sistemas AR de $1^{\mathrm{a}}$ ordem com coeficientes de correlação $\rho$ próximos a unidade [8]. No entanto, para regiões que possuem variações abruptas entre pixels vizinhos como, por exemplo, regiões repletas de bordas, esse modelo não é adequado para estimar a densidade espectral de potência, mas pode servir como indicativo de que a região possui um conteúdo em altas freqüências considerável.

Na Tab.II, estão descritos os passos para adaptação do parâmetro $a$ do filtro de interpolação ${ }_{\left(a=a^{*}\right)} h_{C}$. É importante observar que esse algoritmo corresponde a uma possível implementação de $\left(a=a^{*}\right) h_{C}$ para o caso em que a seqüência de entrada $x_{k}$ é modelada como resposta de um sistema AR de $1^{\mathrm{a}}$ ordem.

\section{RESULTADOS EXPERIMENTAIS}

\section{A. Metodologia}

Com o objetivo de comparar os algoritmos de interpolação quando aplicados a imagens reais, foram realizadas dois diferentes tipos de operações.

- Magnificação: Neste experimento, uma imagem é interpolada por um fator $L$. Na ausência de uma imagem ideal, uma vez que o filtro de reconstrução ideal definido em (4) não é realizável, a imagem interpolada é então 
TABELA II

INTERPOLAÇÃO ADAPTATIVA USANDO FILTROS CÚBICOS.

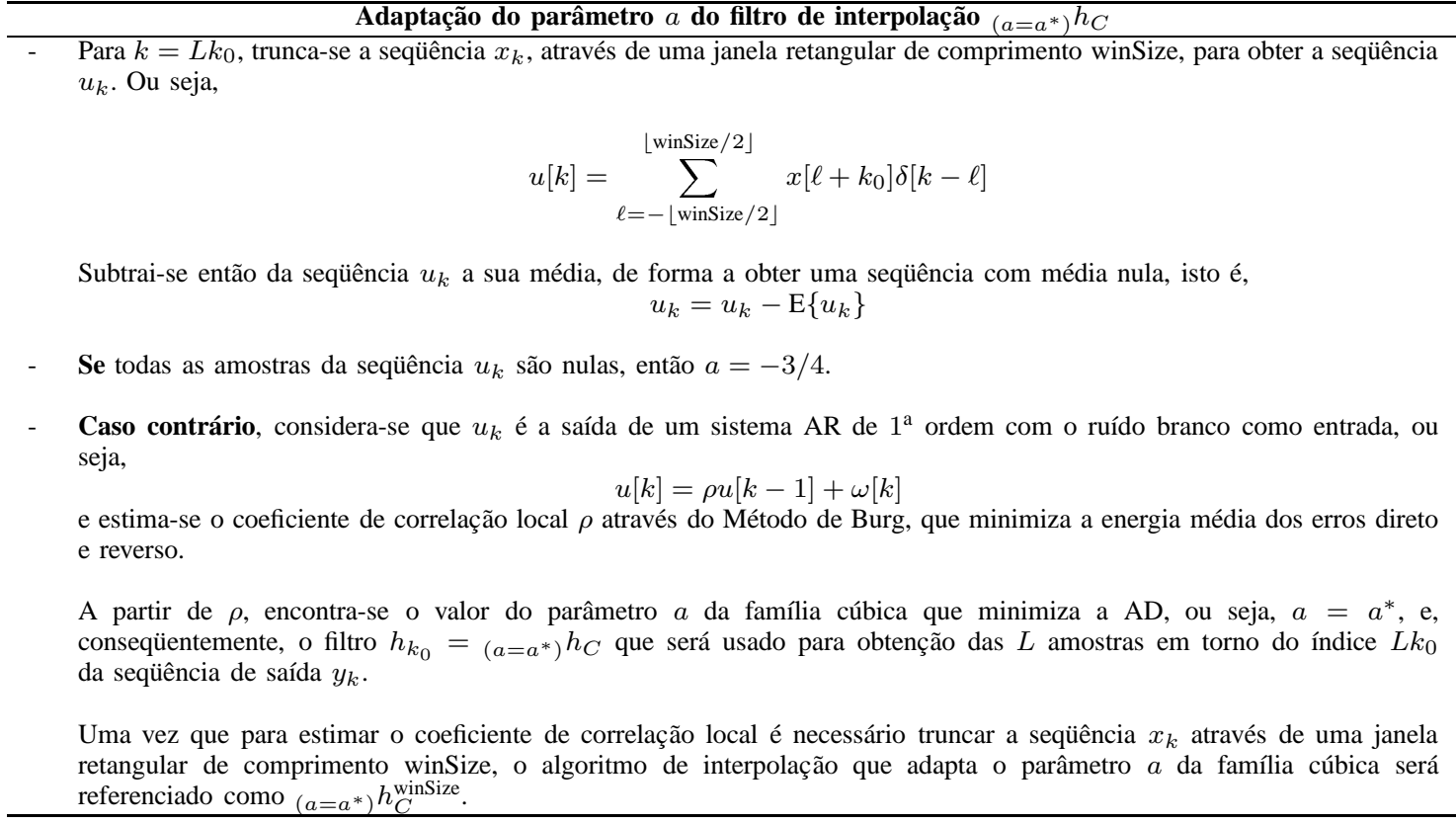

Adaptação do parâmetro $a$ do filtro de interpolação $\left(a=a^{*} h_{C}\right.$

decimada pelo mesmo fator $L$. Portanto, o filtro de decimação é $H_{d}(z)=H_{i}(z) / L$. Veja a Fig. 5.

- Translação: Neste experimento, uma imagem é translatada por um vetor $\overrightarrow{v_{t}}$ para um novo grid. $\mathrm{Na}$ ausência de uma imagem ideal, a imagem translatada é então retranstalada pelo vetor $-\overrightarrow{v_{t}}$, retornando assim ao grid inicial. No final, compara-se as imagens $x_{k}$ e $\hat{x}_{k}$. $\mathrm{O}$ diagrama de blocos do experimento é representado na Fig. 6.

Observe que nos experimentos descritos são necessários dois processos, o direto e o reverso, para obtenção da imagem de saída $\hat{x}_{k}$. E que a imagem desejada é a imagem de entrada $x_{k}$. Essa metodologia possui a vantagem de amplificar o erro de reconstrução inerente aos algoritmos. A medida de distorção utilizada é o Erro Quadrático Médio (MSE - Mean Squared Error), sendo definida em (12).

$$
\operatorname{MSE}=\frac{1}{N_{1} N_{2}} \sum_{k \in \mathbb{Z}^{2}}\left(x_{k}-\hat{x}_{k}\right)^{2}
$$

Para comparar a qualidade visual associada aos filtros de reconstrução, a imagem módulo do erro $e_{k}$, representada nas Figuras 5 e 6, é normalizada da seguinte forma: os pixels com intensidade maior do que $\xi$ são representados em preto; os outros, em branco [6]. Veja (13).

$$
e_{k}=\left\{\begin{aligned}
0 & \text { se }\left|x_{k}-\hat{x}_{k}\right|>\xi \\
255 & \text { se }\left|x_{k}-\hat{x}_{k}\right| \leq \xi
\end{aligned}\right.
$$

onde 0 e 255 representam pixels preto e branco, respectivamente, numa imagem monocromática de 8 bits/pixel.

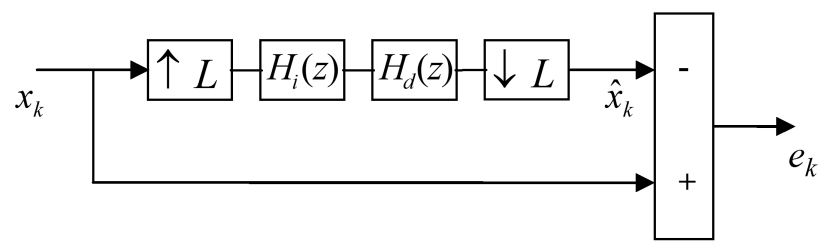

Fig. 5. Experimento de Magnificação por um fator $L$.

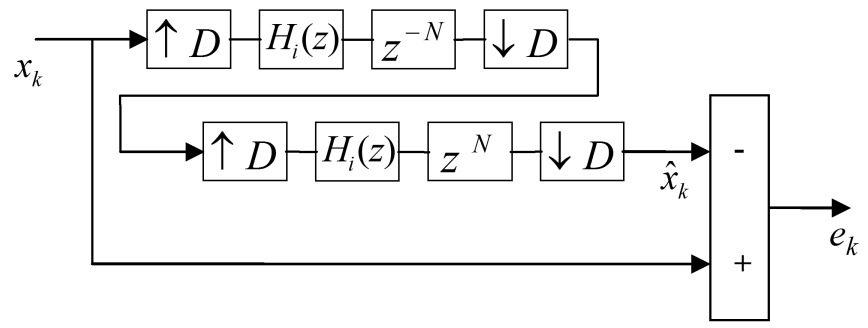

Fig. 6. Experimento de Translação por um vetor $\overrightarrow{v_{t}}=\frac{N}{D}$.

\section{B. Resultados}

Na Fig. 7, estão as imagens monocromáticas que foram utilizadas para avaliar o comportamento dos diversos filtros de reconstrução. As imagens possuem resolução de $512 \times 512$ pixels e 8 bits/pixel. Com o objetivo de eliminar os efeitos de borda, apenas os $472 \times 472$ pixels centrais são considerados no cálculo do MSE.

Nas Tabelas III e IV, estão os resultados associados aos experimentos de magnificação por um fator $L=4$ e translação por um vetor $\overrightarrow{v_{t}}=(.5, .75)$, respectivamente. Foram inseridos nas Tabelas os resultados obtidos pelos filtros de reconstrução de ordem zero $\left(h_{N}\right)$, ou Método do Vizinho Mais Próximo, linear $\left(h_{L}\right)$ e quadrático $\left(h_{Q}\right)$. Este último, proposto por 


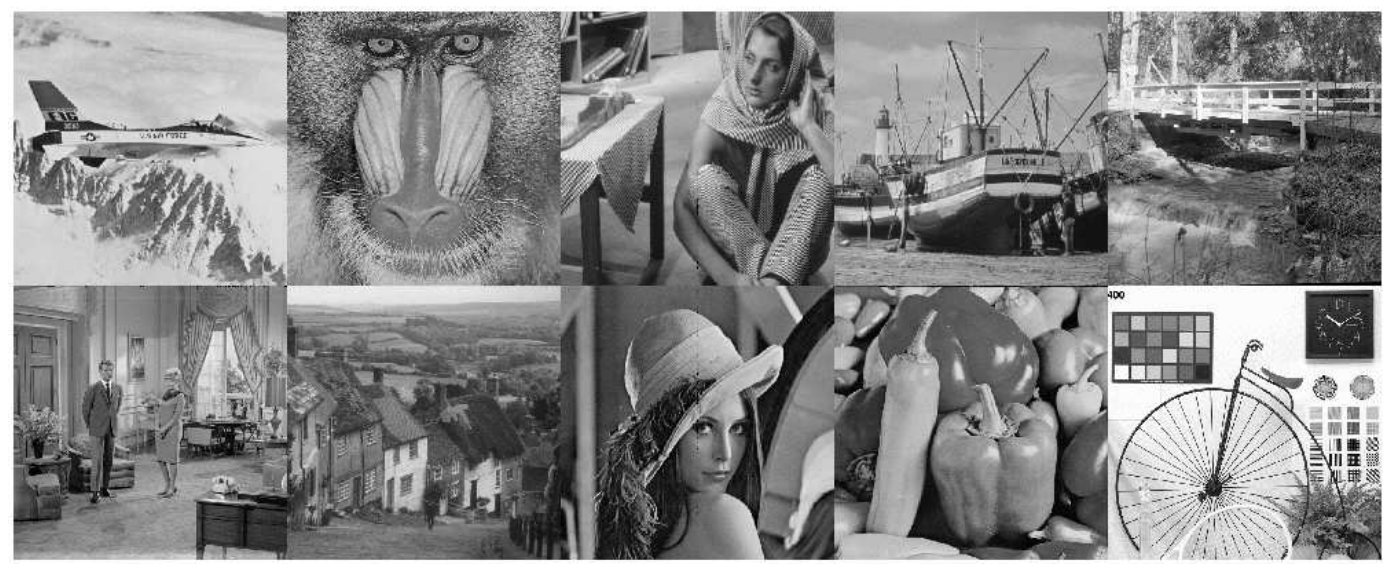

Fig. 7. Imagens teste com resolução de $512 \times 512$ pixels e 8 bits/pixel.

Dogdson em [9].

Observe nas Tabelas III e IV que, dentre os algoritmos clássicos da família cúbica, os melhores resultados são obtidos quando o parâmetro $a$ assume os valores $-3 / 4$ e -1 . Para imagens repletas de bordas, como as imagens Barbara e Watch, $a=-1$ mostrou ser o valor mais adequado em virtude da maior concentração da energia em baixas freqüências. Já para imagens repletas de regiões de textura, $a=-3 / 4$ mostrou ser o valor mais adequado. No entanto, para todas as imagens, a adaptação do parâmetro $a$ da família cúbica apresentou um ganho significativo em relação aos algoritmos clássicos.

$\mathrm{Na}$ Fig 8, estão as imagens erro associadas aos filtros de interpolação ${ }_{(a=-3 / 4)} h_{C}, \quad(a=-1) h_{C}$ e ${ }_{\left(a=a^{*}\right)} h_{C}^{11}$ para o experimento de Magnificação por um fator $L=4$. Observe que, para $\xi=10$, a imagem erro do algoritmo adaptativo possui baixa densidade de pixels pretos.

\section{Conclus Ão}

Nos algoritmos clássicos de interpolação, o valor do parâmetro $a$ da família cúbica é constante. Com o objetivo de minimizar o erro inerente a qualquer algoritmo de interpolação realizável, foi proposto um algoritmo que adapta o parâmetro $a$ de acordo com propriedades locais da seqüência a ser interpolada.

Uma possível implementação do algoritmo adaptativo para a classe de seqüências que resultam de um sistema AR de $1^{\mathrm{a}}$ ordem foi descrita. Simulações em imagens típicas mostram um ganho quantitativo significativo do algoritmo adaptativo quando comparado com algoritmos clássicos.

\section{REFERÊNCIAS}

[1] R. Bernstein, "Digital Image Processing of Earth Observation Sensor Data". IBM Jornal of Research Development, pp. 40-57. January, 1976.

[2] R. G. Keys, "Cubic Convolution Interpolation for Digital Image Processing", IEEE Transactions on Acoustics, Speech and Signal Processing, vol. ASSP, pp. 1153-1160, 1981.

[3] J. A. Parker, R. V. Kenyon e D. E. Troxel, "Comparison of interpolation methods for image resampling", IEEE Transactions on Medical Imaging, Vol. MI-2, No. 1, pp. 31-39, 1983.

[4] E. Maeland, "On The Comparison Of Interpolation Methods", IEEE Transactions on Medical Imaging, Vol. 7, No. 3, September 1988.
[5] E. H. W. Meijering, K. J. Zuiderveld e M. A. Viergever, "Image Reconstruction by Convolution with Symmetrical Piecewise $n$ th-Order Polynomial Kernels", IEEE Transactions on Image Processing, Vol. 8, No. 2, February 1999.

[6] T. Lehmann, C. Gönner e K. Spitzer, "Survey: Interpolation Methods in Medical Image Processing", IEEE Transactions on Medical Imaging, Vol. 18, November 1999.

[7] P. Thévenaz, T. Blu e M. Unser, "Interpolation Revisited", IEEE Transactions on Medical Imaging, 2000.

[8] N. S. Jayant e P. Noll, "Digital Coding of Waveforms", Prentice-Hall Signal Processing Series, Englewood Cliffs, NJ, USA: Prentice-Hall Inc., 1984.

[9] N. A. Dogdson, "Quadratic Interpolation for Image Resampling", IEEE Transactions on Image Processing, Vol. 6, pp. 1322-1326, 1997.

[10] R. Suntheralingam, "Adaptive Cubic Convolution of High Resolution Remotely Sensed Image Data", Asian Conference on Remote Sensing, 1995.

[11] J. Han e S. Baek, "Parametric Cubic Convolution Scaler for Enlargement and Reduction of Image", IEEE Transactions on Consumer Eletronics, Vol. 46, No. 2, pp. 247-256, 2000.

[12] J. Han e H. Kim, "Modified Cubic Convolution Scaler with Minimum Loss of Information", Society of Photo-Optical Instrumentation Engineers, 2001.

[13] S. E. Khamy, M. M. Hadhoud, M. I. Dessouky, B. M. Salem e F. E. Samie, "An Adaptive Cubic Convolution Image Interpolation Approach", Machine Graphics \& Vision International Journal, Vol. 14, 2005 . 
TABELA III

MSE dos ALGORITMOS DE INTERPOLAÇÃo ASSOCIAdOS À OPERAÇÃo DE MAGNIFICAÇÃo POR UM FATOR $L=4$.

\begin{tabular}{c|r|r|r|r|r|r|r|r|r|r}
\hline \multirow{2}{*}{$\begin{array}{c}\text { Filtro de } \\
\text { Interpolação }\end{array}$} & \multicolumn{10}{|c}{ Imagem } \\
\cline { 2 - 13 } & Airplane & Baboon & Barbara & Boat & Bridge & Couple & Goldhill & Lena & Peppers & Watch \\
\hline$h_{N}$ & 16.37 & 80.67 & 65.11 & 22.58 & 49.95 & 26.77 & 18.75 & 21.25 & 14.37 & 166.09 \\
\hline$h_{L}$ & 8.64 & 80.87 & 60.79 & 14.45 & 43.46 & 20.09 & 14.92 & 12.42 & 10.09 & 161.05 \\
\hline$h_{Q}$ & 4.17 & 43.01 & 31.69 & 7.22 & 23.06 & 10.18 & 7.80 & 6.36 & 5.61 & 85.61 \\
\hline$(a=0) h_{C}$ & 6.00 & 56.99 & 42.83 & 10.02 & 30.69 & 13.94 & 10.44 & 8.78 & 7.29 & 113.52 \\
$(a=-1 / 2) h_{C}$ & 1.86 & 29.32 & 17.89 & 3.97 & 15.40 & 6.17 & 5.17 & 3.57 & 4.15 & 57.90 \\
$(a=-3 / 4) h_{C}$ & 1.29 & 20.97 & 9.75 & 2.63 & 11.16 & 4.39 & 3.73 & 2.60 & 3.35 & 41.16 \\
$(a=-1) h_{C}$ & 2.31 & 19.87 & 8.00 & 3.18 & 11.20 & 4.95 & 3.74 & 3.49 & 3.45 & 38.27 \\
$\left(a=a^{*}\right) h_{C}^{11}$ & 1.26 & 19.12 & 4.93 & 2.46 & 10.66 & 4.29 & 3.52 & 2.48 & 3.22 & 34.54 \\
\hline
\end{tabular}

TABELA IV

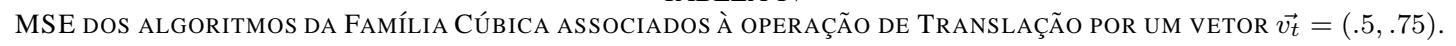

\begin{tabular}{|c|c|c|c|c|c|c|c|c|c|c|}
\hline \multirow{2}{*}{$\begin{array}{c}\text { Filtro de } \\
\text { Interpolação }\end{array}$} & \multicolumn{10}{|c|}{ Imagem } \\
\hline & Airplane & Baboon & Barbara & Boat & Bridge & Couple & Goldhill & Lena & Peppers & Watch \\
\hline$h_{N}$ & 137.73 & 818.08 & 258.55 & 120.92 & 422.82 & 195.10 & 139.09 & 108.16 & 100.25 & 1304.96 \\
\hline$h_{L}$ & 16.43 & 161.60 & 93.11 & 23.70 & 80.10 & 37.22 & 26.86 & 21.02 & 17.34 & 290.96 \\
\hline$h_{Q}$ & 11.81 & 139.73 & 47.97 & 12.78 & 62.19 & 26.41 & 19.17 & 13.50 & 13.53 & 218.50 \\
\hline$(a=0) h_{C}$ & 13.63 & 148.20 & 63.54 & 16.59 & 68.62 & 30.25 & 21.80 & 16.36 & 14.93 & 243.76 \\
\hline$(a=-1 / 2) h_{C}$ & 4.30 & 82.91 & 24.17 & 5.96 & 35.31 & 13.92 & 10.84 & 6.86 & 8.52 & 128.51 \\
\hline$(a=-3 / 4)^{h}$ & 3.07 & 62.96 & 12.48 & 4.03 & 26.48 & 10.21 & 8.02 & 5.14 & 6.96 & 94.41 \\
\hline$(a=-1) h_{C}$ & 5.43 & 60.11 & 13.18 & 5.80 & 27.24 & 11.44 & 8.40 & 7.06 & 7.31 & 87.96 \\
\hline$\left(a=a^{*}\right) h_{C}^{11}$ & 3.01 & 57.11 & 8.64 & 3.84 & 25.71 & 10.07 & 7.59 & 4.89 & 6.79 & 80.15 \\
\hline
\end{tabular}
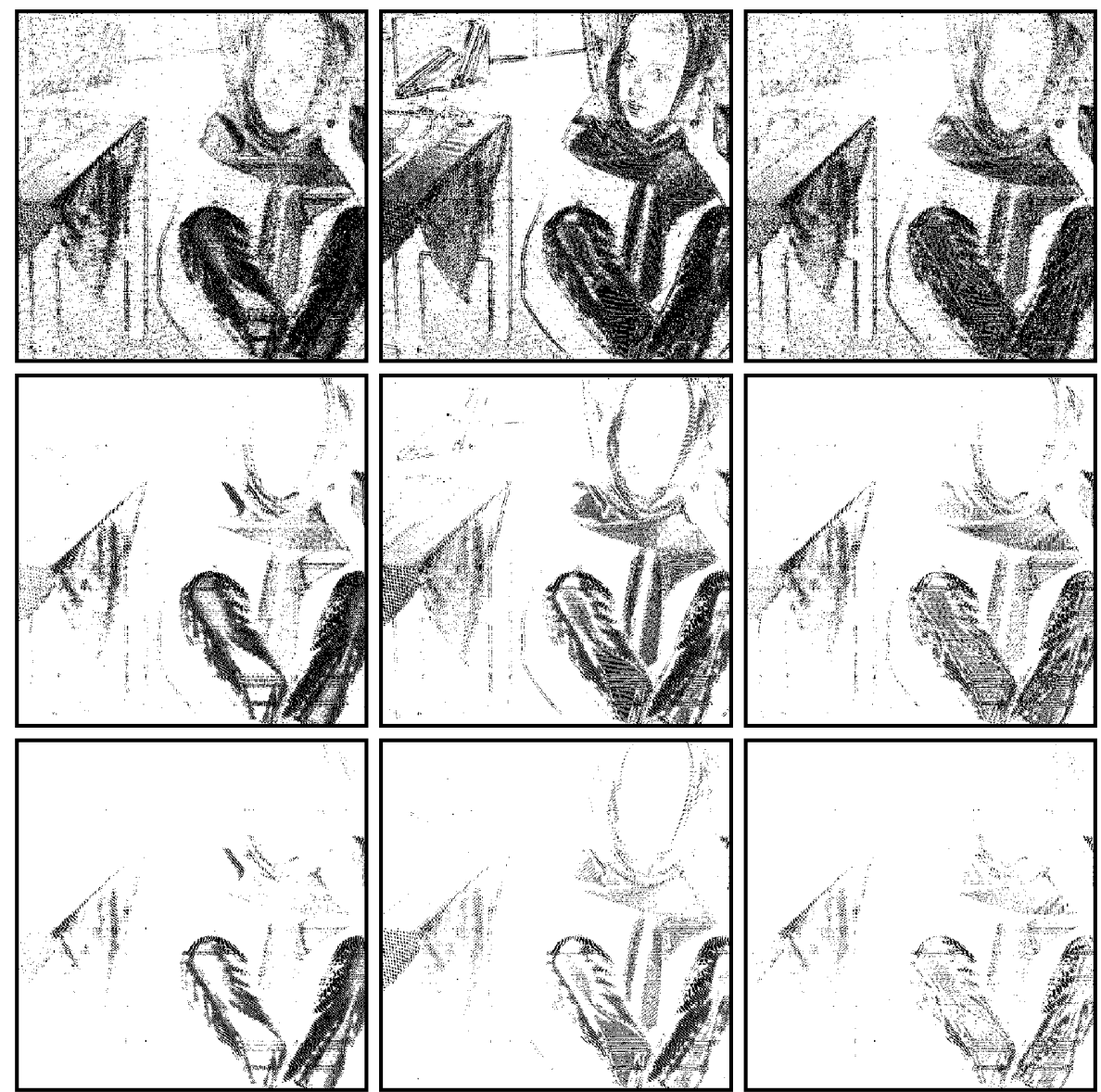

Fig. 8. Imagens erro para o experimento de Magnificação por um fator $L=4$. Da esquerda para a direita, seguem as imagens erro obtidas a partir dos filtros de interpolação ${ }_{(a=-3 / 4)} h_{C},{ }_{(a=-1)} h_{C} \mathrm{e}_{\left(a=a^{*}\right)} h_{C}^{11}$ para $\xi=1,5$ e 10 , respectivamente. 